\title{
ESTUDO CITOFOTOMÉTRICO DA EXPRESSÃO DO MARCADOR TUMORAL FATOR VIII E FATORES PROGNÓSTICOS NO ADENOCARCINOMA GÁSTRICO
}

\section{Cytophotometric study of the expression of the tumoral marker Factor VIII and prognostic factors in gastrci adenocarcinoma}

\author{
Mary Tossa NAKAMURA ${ }^{1}$, Osvaldo MALAFAIA ${ }^{2}$, Ronaldo Mafia CUENCA $^{1}$, \\ Nicolau Gregori CZECZKO ${ }^{2}$, Regina Maria da CUNHA $^{2}$, Teresa Cristina Santos CAVALCANTI ${ }^{2}$, \\ Carmen Austrália Paredes Marcondes RIBAS ${ }^{2}$, Alexandre Eduardo Augustin CZECZKO $^{2}$
}

ABCDDV/566

Nakamura MT, Malafaia O, Cuenca RM, Czeczko NG, Cunha RM, Cavalcanti TCS, Ribas CAPM, Czeczko AEA. Estudo citofotométrico da expressão do marcador tumoral Fator VIII e fatores prognósticos no adenocarcinoma gástrico. ABCD Arq Bras Cir Dig 2007;20(4):234-40.

RESUMO - Racional - No câncer gástrico, a incidência, o diagnóstico e as opções terapêuticas apresentaram melhorias nas últimas décadas, porém o prognóstico permanece reservado, especialmente devido à maioria dos pacientes procurarem recurso médico com tumores avançados, metastáticos ou cirurgicamente irressecáveis já no diagnóstico. Biologia molecular é área de conhecimento recente com grandes questionamentos a serem respondidos e a atualidade dos fatos científicos mostra que o caminho deverá ser através da identificação de marcadores tumorais. Os grandes avanços na área da informática aprimoraram a análise da imagem celular através da citofotometria de imagem que possibilita através da imunoistoquímica estudar a proliferação celular e a angiogênese que participa em diversos processos tumorais, sendo pesquisadas por vários marcadores. Atualmente estudos são realizados para demonstrar o valor prognóstico de suas expressões, contudo, no adenocarcinoma gástrico resultados têm sido divergentes e estudos escassos. Objetivos - Identificar e quantificar citofotometricamente a expressão dos marcadores da angiogênese através do fator VIII no adenocarcinoma e comparar suas expressões com fa classificação de Bormann, profundidade de invasão tumoral, grau de diferenciação, envolvimento nodal, padrão histológico e idade. Métodos - Foram estudados 21 pacientes com adenocarcinoma gástrico, identificados de 1998 a 2006. Para a deteç̧ão do Fator VIII foi realizada imunoistoquímica, com anticorpo policlonal para Fator VIII. Foi realizada análise citofotométrica informatizada pelo sistema SAMBA 4000. Resultados - Dos 21 pacientes 61,90\% eram do sexo masculino e 38,10\% do feminino, com idade mediana de 65 anos e apenas um não marcou para o Fator VIII (95,24\% de marcação). As médias dos índices de marcagem para o Fator VIII foram de 61,14\% (desvio-padrão de 15,06, variando de 29,16 a 73,91). Tumores com classificação III ou IV apresentaram índice de marcagem maiores do que aqueles com Bormann I ou II, porém sem correlação com a profundidade de invasão tumoral, grau de diferenciação, envolvimento nodal e padrão histológico. Conclusões - O presente estudo identificou e marcou 95,24\% das amostras para o Fator VIII. Em relação aos fatores prognósticos não houve correlação significativa exceto entre o Fator VIII e a classificação de Bormann no qual o tipo III ou IV foi maior que o tipo I ou II.

DESCRITORES - Adenocarcinoma gástrico. Fator VIII. Citofotometria de imagem. SAMBA.

\section{INTRODUÇÃO}

Apesar do declínio na incidência em muitas nações industrializadas, o câncer gástrico permanece como maior problema de saúde e a segunda causa de morte por neoplasia maligna no mundo, sendo a prevenção a maior estratégia promissora de controle da doença ${ }^{3}$. A incidência, o diagnóstico e as opções terapêuticas apresentaram melhorias nas últimas décadas, porém o prognóstico permanece reservado, especialmente devido à maioria dos pacientes procurarem recurso médico com tumores avançados, metastáticos ou cirurgicamente irressecáveis já no diagnóstico ${ }^{2}$. A doença é freqüente no Brasil, ocupando a quinta colocação, com diferenças regionais.

$\mathrm{O}$ adenocarcinoma é o tipo mais comum de neoplasia maligna do estômago, correspondendo à aproximada-

Trabalho realizado na ${ }^{1}$ União Educacional do Planalto Central - UNIPLAC - Brasília, DF e ${ }^{2}$ Hospital Universitário Evangélico de Curitiba - HUEC - Curitiba, PR, Brasil.

Endereço para correspondência: Ronaldo Mafia Cuenca, e-mail: rmcuenca@uol.com.br mente 90 a $95 \%$ de todos os $\operatorname{casos}^{4}$. Os tumores nãoepiteliais constituem $5 \%$ e os mais freqüentes são os linfomas e os leiomiossarcomas.

O estudo anatomopatológico e o estadiamento pré-operatório são importantes para o planejamento diagnóstico e terapêutico. Histologicamente, o carcinoma gástrico pode ser classificado em dois tipos: o intestinal e o difuso ${ }^{12}$. O tipo intestinal relaciona-se com epitélio metaplásico e com formação de tumores volumosos. O tipo difuso apresentase com células pouco diferenciadas infiltrando o estroma gástrico e freqüentemente com crescimento infiltrativo conhecido como linite plástica de pior prognóstico, que acomete pacientes mais jovens ${ }^{16}$. No estadiamento existem dois sistemas: o proposto pela International Union Against Cancer (UICC) e pela Japanese Gastric Cancer Association (JGCA). Ambos utilizam como parâmetro a profundidade de invasão de parede gástrica $(\mathrm{T})$, a presença de metástase linfonodal $(\mathrm{N})$ e a ocorrência de metástase à distância $(\mathrm{M})^{17}$.

Excluindo câncer gástrico precoce no Japão (com taxa de sobrevida em cinco anos de aproximadamente $95 \%{ }^{11}$ ), o 
prognóstico não tem melhorado nos últimos anos e a sobrevivência em cinco anos tem sido estimada em apenas $40 \%$, e naqueles com ressecção completa a taxa de recorrência apresenta-se muito alta. A sobrevida pode ser melhorada com a quimioradioterapia pós-operatória. Apesar de grande desenvolvimento quanto ao regime quimioterápico, mais efetivo e menos tóxica, modesta eficácia tem sido demonstrada na doença metastática ${ }^{13,18}$.

Estudos bem conduzidos com caracterização patológica e identificação de marcadores tumorais têm permitido a evolução na cirurgia, na patologia e no tratamento coadjuvante do câncer gástrico. Contudo, entendimento maior nas alterações moleculares é necessário ${ }^{5}$.

Biologia molecular é área de conhecimento recente com grandes questionamentos a serem respondidos, estimando-se algumas décadas à frente para se ter confirmação das idéias hoje existentes. A atualidade dos fatos científicos mostra que o caminho deverá ser através de detecção de marcadores que entram no ciclo celular como estimuladores do desenvolvimento celular, inibidores da mitose, alterações moleculares do DNA obtidas por interferências externas e fatores de crescimento, hormônios esteróides, dentre outros.

Mostram-se promissores na detecção dos agentes que interferem na multiplicação e fenótipos celulares, marcadores de proteínas que atuam no ciclo celular. Vários métodos têm sido tentados com sucesso para obter este objetivo. A maior dificuldade é a necessidade de tecnologia e operacionalidade de alto custo tanto em material quanto em equipamentos. A virtualidade da informática tem sido utilizada para minorar estes inconvenientes em função de que, com o uso de softwares que não ocupam espaço e são de custo previsível, a marcação destas proteínas poderia ser quantificada e qualificada com maior facilidade e menor custo.

Os grandes avanços na área da informática aprimoraram a análise da imagem celular através da citometria de imagem ou citofotometria que possibilita através da imunoistoquímica marcar as desejadas interferências supostas como fatores etiopatogênicos das doenças. Vários marcadores estão sendo testados, entre os quais o Fator VIII, que permite coloração das estruturas transformando as áreas marcadas em imagens numéricas que são quantificadas pelo computador.

A proliferação desordenada de um tecido ou célula depende do desequíbrio entre os fatores bloqueadores e estimuladores do ciclo celular, principalmente na fase de síntese ou fase S. Várias são as proteínas que atuam pró ou contra a multiplicação, somando-se a ela ainda a ação de fatores de crescimento e de apoptose.

Angiogênese é o processo no qual ocorre o aumento proporcional da microvascularização em uma estrutura ou tecido. Ele é muito controlado e dependente de inúmeros fatores estimuladores e inibidores. Os recentes avanços na terapia do câncer estão relacionados em parte ao desenvolvimento de agentes antiangiogênicos, que sinalizam serem elementos de investigação, controle e tratamento na perspectiva de progresso na luta contra o câncer e suas metástases?

Existem vários marcadores que lutam entre si para serem reconhecidos como o melhor dentre os demais para marcar a neovascularização característica da angiogênese. Dentre eles está o Fator VIII. Muitas evidências sugerem que o estudo da angiogênese possa provar um novo conceito de prognóstico em pacientes com câncer gastrointestinal, porém resultados de estudos retrospectivos necessitam serem validados por estudos prospectivos ${ }^{15}$.

Os objetivos deste estudo foram: identificar e quantificar citofotometricamente a expressão do marcador de angiogênese Fator VIII e comparar a expressão dele com fatores prognósticos: profundidade de invasão tumoral, grau de diferenciação, envolvimento nodal, padrão histológico e idade.

\section{MÉTODOS}

A amostra inicial consistiu de 55 blocos parafinados e fixados em formalina de peças advindas de gastrectomias com adenocarcinoma gástrico. O material foi proveniente dos serviços de patologia do Hospital Regional do Gama do Distrito Federal e do Hospital Dom Arione de Araguaina (Tocantins), de pacientes submetidos a ressecções gástricas no período de 1998 a 2006 nos serviços de cirurgia e de oncologia dos hospitais mencionados. Cada amostra foi pré-selecionada de acordo com o estado de conservação, confecção e que representavam melhor a massa tumoral. Os critérios de inclusão foram amostras com confirmação de adenocarcinoma gástrico pela coloração hematoxilinaeosina e os de exclusão amostras que não apresentavam concordância com o diagnóstico, material inadequado (tecido necrosado, fixação inadequada, formol não tamponado, tecido dobrado ou com sobreposição), artefatos, marcagem inadequada e não reação adequada à imunoistoquímica. Os espécimes estavam identificados e processados com as técnicas histológicas de rotina, conservados em solução de formol tamponado.

Os blocos de parafina foram submetidos ao preparo habitual para técnica imunoistoquímica e utilizou-se o reagente anti-Fator VIII, anticorpo anti-humano, policlonal de camundongo.

O sistema de análise citofotométrica utilizado foi o sistema SAMBA 4000 - Systéme d'Analyse Microphotometrique á Balayage Automatique (Sistema de Análise Microscópica de Busca Automática) -, desenvolvido pela Alcatel (Grenoble, França). Ele é composto por microscópio com sistema de iluminação, vídeocâmera, microcomputador capaz de captar imagens microscópicas, impressora, e um software com capacidade para interpretar e analisar matematicamente as imagens por ele captadas chamado Immuno criado para analisar três variáveis que caracterizam quantitativamente as reações de coloração histoquímica: índice de marcagem, densidade óptica média e heterogeneidade de concentração.

$\mathrm{O}$ índice de marcagem descreve a quantidade em percentagem da área tecidual que foi corada em relação lâmina como um todo.

A densidade óptica média descreve a intensidade de coloração demonstrando que áreas da lâmina apresentam maior densidade de cor, maior densidade ótica da reação imunológica e conseqüentemente, maior expressão do marcador utilizado.

Aheterogeneidade de concentração expressa a distribuição da 
concentração do marcador nos campos individuais avaliados.

A representação das diferentes variáveis calculadas pela quantificação do marcador imunoistoquimico, através da imagem computadorizada permite, em um mesmo momento, observar a área da superfície analisada, a intensidade de marcação e a concentração da coloração.

A análise estatística foi descrita por médias, medianas, valores mínimos, valores máximos e desvios-padrão. A comparação entre grupos definidos por profundidade de invasão tumoral (T1/T2 ou T3/T4), classificação (tipo Bormann I/II ou III/IV), tipo (pouco diferenciado ou moderado/ bem diferenciado), envolvimento nodal (N0 ou N1/N2/N3), idade (até 65 anos ou mais de 65 anos), histologia (intestino ou difuso), foi feita usando-se o teste não-paramétrico de Mann-Whitney. A condição de normalidade dos dados foi verificada pelo teste de Kolmogorov-Smirnov. Valores de $\mathrm{P}<0,05$ indicaram significância estatística.

\section{RESULTADOS}

Da amostra inicial de 55 blocos (pacientes) com adenocarcinoma gástrico identificados em período de 8 anos (1998 a 2006), após serem aplicados os critérios de exclusão, 21 pacientes foram considerados elegíveis para a análise deste estudo (Figura 1). Deles, a expressão do marcador Fator VIII foi obtida em 20 pacientes (Tabelas 1 e 2).

A idade média foi de 62,33 anos, mediana de 65 anos,

TABELA 1 - Análise histopatológica dos tumores gástricos examinados

\begin{tabular}{|c|c|c|c|c|c|c|c|}
\hline OBS & $\begin{array}{c}\text { Padrão } \\
\text { histológico }\end{array}$ & Classificação & $\begin{array}{l}\text { Profundidade de } \\
\text { invasão tumoral }\end{array}$ & $\begin{array}{c}\text { Grau de } \\
\text { diferenciação }\end{array}$ & $\begin{array}{c}\text { Envolvimento } \\
\text { nodal }\end{array}$ & Sexo & Idade \\
\hline 1 & $\mathrm{D}$ & B.III & $\mathrm{T} 3$ & POUCO & No & $\mathrm{M}$ & 59 \\
\hline 2 & I & B. III & $\mathrm{T} 3$ & MOD. & N1 & M & 69 \\
\hline 3 & D & B. III & $\mathrm{T} 3$ & MOD. & N1 & M & 69 \\
\hline 4 & I & B. III & $\mathrm{T} 2$ & MOD. & No & M & 57 \\
\hline 5 & D & B. IV & $\mathrm{T} 4$ & POUCO & N3 & F & 50 \\
\hline 6 & I & B. III & $\mathrm{T} 3$ & MOD. & N1 & $\mathrm{F}$ & 61 \\
\hline 7 & D & B. IV & $\mathrm{T} 3$ & POUCO & N1 & F & 77 \\
\hline 8 & D & B. II & $\mathrm{T} 2$ & MOD. & No & M & 41 \\
\hline 9 & I & B. II & $\mathrm{T} 2$ & MOD. & N1 & M & 70 \\
\hline 10 & I & B. II & $\mathrm{T} 2$ & MOD. & N1 & M & 59 \\
\hline 11 & I & B. II & $\mathrm{T} 1$ & MOD. & No & F & 76 \\
\hline 12 & I & B. III & $\mathrm{T} 4$ & MOD. & N2 & M & 77 \\
\hline 13 & D & B. II & $\mathrm{T} 4$ & BEM & N1 & $\mathrm{F}$ & 73 \\
\hline 14 & D & B. III & $\mathrm{T} 4$ & POUCO & N3 & M & 49 \\
\hline 15 & D & B. I & $\mathrm{T} 3$ & INDEF. & N1 & M & 70 \\
\hline 16 & I & B. I & $\mathrm{T} 2$ & BEM & No & F & 56 \\
\hline 17 & D & B. III & $\mathrm{T} 3$ & BEM & No & M & 40 \\
\hline 18 & D & B. II & $\mathrm{T} 2$ & POUCO & No & $\mathrm{F}$ & 73 \\
\hline 19 & D & B. II & $\mathrm{T} 4$ & POUCO & N3 & F & 43 \\
\hline 20 & I & B. II & T3 & MOD. & N1 & M & 65 \\
\hline
\end{tabular}

$\mathrm{I}=$ Intestinal $/ \mathrm{D}=$ Difuso $/ \mathrm{B}=$ Bormann $/ \mathrm{MOD}$. = Moderadamente diferenciado; POUCO $=$ Pouco diferenciado $/$ BEM $=$ Bem diferenciado $/$ INDIF.$=$ Indiferenciado $\mathrm{M}=$ Masculino $; \mathrm{F}=$ Feminino

variando de 40 a 77 anos, com desvio-padrão de 12,18 anos, sendo 13 pacientes do sexo masculino $(61,90 \%)$ e oito do feminino $(38,10 \%)$.

\section{Classificação de Bormann}

TABELA 2 - Análise citofotométrica pelo marcador Fator VIII

\begin{tabular}{ccccccc}
\hline OBS Código & $\begin{array}{c}\text { Número } \\
\text { exame }\end{array}$ & $\begin{array}{c}\text { Campos } \\
\text { lidos }\end{array}$ & $\begin{array}{c}\text { Índice de } \\
\text { Marcagem }\end{array}$ & Heterogeneidade & $\begin{array}{c}\text { Densidade } \\
\text { óptica }\end{array}$ \\
\hline 1 & A2 & $3385-03$ & 10 & $72,61 \%$ & $0,32 \%$ & 69,63 \\
2 & A4 & $3077-01$ & 2 & $74,11 \%$ & $0,29 \%$ & 60,29 \\
3 & A5 & $7186-01$ & 10 & $75,71 \%$ & $0,29 \%$ & 62,72 \\
4 & A6 & $3337-04$ & 10 & $72,81 \%$ & $0,31 \%$ & 69,89 \\
5 & A7 & $1824-03$ & 10 & $71,92 \%$ & $0,32 \%$ & 61,72 \\
6 & A9 & $6904-03$ & 10 & $57,50 \%$ & $0,35 \%$ & 63,62 \\
7 & A10 & $1216-04$ & 10 & $67,18 \%$ & $0,28 \%$ & 61,99 \\
8 & A12 & $143-05$ & 10 & $71,21 \%$ & $0,33 \%$ & 72,73 \\
9 & A13 & $8940-03$ & 10 & $71,20 \%$ & $0,3 \%$ & 67,34 \\
10 & A15 & $522-05$ & 10 & $71,33 \%$ & $0,25 \%$ & 65,71 \\
11 & A18 & $3246-02$ & 10 & $69,15 \%$ & $0,33 \%$ & 71,27 \\
12 & A19 & $1056-02$ & 10 & $73,91 \%$ & $0,29 \%$ & 67,19 \\
13 & A20 & $1094-02$ & 10 & $72,10 \%$ & $0,29 \%$ & 67,44 \\
14 & A21 & $1727-0$ & 10 & $72,12 \%$ & $0,3 \%$ & 62,61 \\
15 & A22 & $0003-01$ & 10 & $61,75 \%$ & $0,33 \%$ & 60,93 \\
16 & A24 & $2884-01$ & 10 & $48,87 \%$ & $0,33 \%$ & 51,29 \\
17 & A25 & $165-02$ & 10 & $50,79 \%$ & $0,29 \%$ & 55,60 \\
18 & A26 & $1216-4$ & 10 & $40,55 \%$ & $0,33 \%$ & 46,37 \\
19 & A30 & $2465-00$ & 10 & $51,66 \%$ & $0,28 \%$ & 54,43 \\
20 & A37 & $675-99$ & 10 & $29,16 \%$ & $0,32 \%$ & 48,68 \\
\hline
\end{tabular}

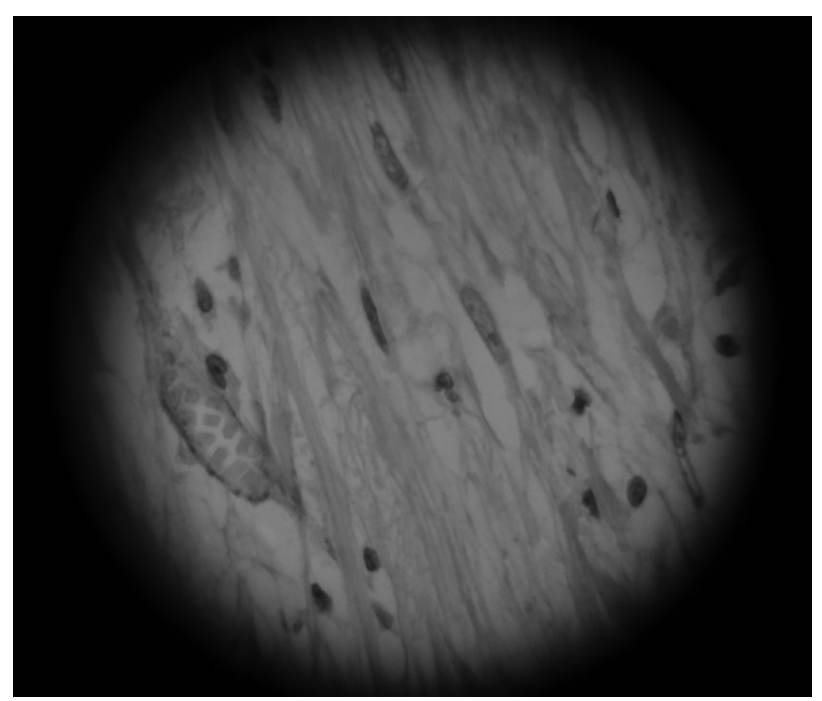

FIGURA 1 - Imunorreatividade do Fator VIII nas células de vasos angiogênicos (aumento de 400x)

Em relação a classificação de Bormann, testou-se a hipótese nula de que tumores com classificação Bormann I e II têm resultados iguais a tumores com classificação Bormann III e IV versus a alternativa de resultados diferentes. Na Tabela 3 estão apresentados os valores de média, mediana, mínimo, máximo e desvio-padrão obtidos em relação à ela.

Os resultados dos testes estatísticos indicaram que não há diferença significativa entre tumores com classificação Bormann I e II versus III e IV (Tabela 3). Já em relação ao índice de marcagem do Fator VIII, o resultado do teste indicou a rejeição da hipótese nula $(\mathrm{P}=0,019)$ onde tumores com classificação Bormann III e IV apresentam índice de marcagem maiores do que tumores com classificação 
TABELA 3 - Expressão do Fator VIII e a classificação de Bormann

\begin{tabular}{ccccccccc}
\hline Fator VIII & Classificação & n & Média & Mediana & Mínimo & Máximo & $\begin{array}{c}\text { Desvio- } \\
\text { padrão }\end{array}$ & $\begin{array}{c}\text { Valor } \\
\text { de } \boldsymbol{P}\end{array}$ \\
\hline $\begin{array}{c}\text { Índice de } \\
\text { marcagem }\end{array}$ & Bormann I ou II & 10 & 58,70 & 65,45 & 29,16 & 72,10 & 15,34 & 0,019 \\
Bermann III ou IV & 10 & 68,87 & 72,37 & 50,79 & 75,71 & 8,22 & \\
$\begin{array}{c}\text { Densidade } \\
\text { óptica }\end{array}$ & Bormann I ou II & 10 & 60,62 & 63,32 & 46,37 & 72,73 & 9,71 & 0,739 \\
$\begin{array}{c}\text { Heteroge- } \\
\text { neidade }\end{array}$ & Bormann III ou IV & 10 & 63,53 & 62,67 & 55,60 & 69,89 & 4,37 & \\
\hline
\end{tabular}

Bormann I e II. Desta forma, pode-se afirmar que existe diferença significativa entre as classificações de Bormann I ou II e Bormann III ou IV em relação ao índice de marcagem do Fator VIII.

\section{Profundidade de invasão tumoral}

Para cada uma das expressões do marcador, testouse a hipótese nula de que tumores com profundidade de invasão tumoral T1 e T2 têm resultados iguais a tumores com profundidade de invasão tumoral T3 e T4, versus a alternativa de resultados diferentes. Na Tabela 4 são apresentados os valores de média, mediana, mínimo, máximo e desvio-padrão obtidos para tumores com profundidade de invasão tumoral T1 e T2 e com os de T3 e T4.

Os resultados dos testes estatísticos indicaram que não há diferença significativa entre tumores com profundidade de invasão tumoral T1 e T2 com os de T3 e T4, em relação aos índices de marcagem, densidade óptica e heterogeneidade (Tabela 4).

\section{Grau de diferenciação tumoral}

TABELA 4 - Expressão do Fator VIII e a profundidade de invasão tumoral

\begin{tabular}{cccccccccc}
\hline Fator VIII Prof. inv. tumoral & $\mathbf{n}$ & Média & Mediana & Mínimo & Máximo & $\begin{array}{c}\text { Desvio- } \\
\text { padrão }\end{array}$ & $\begin{array}{c}\text { Valor } \\
\text { de } \boldsymbol{P}\end{array}$ \\
\hline $\begin{array}{c}\text { Índice de } \\
\text { marcagem }\end{array}$ & T1 ou T2 & 7 & 63,59 & 71,20 & 40,55 & 72,81 & 13,16 & 0,485 \\
& T3 ou T4 & 13 & 63,89 & 71,92 & 29,16 & 75,71 & 13,55 & \\
$\begin{array}{c}\text { Densidade } \\
\text { óptica }\end{array}$ & T1 ou T2 & 7 & 63,51 & 67,34 & 46,37 & 72,73 & 10,40 & \\
Heteroge- & T3 ou T4 & 13 & 61,30 & 61,99 & 48,68 & 69,63 & 5,71 & 0,241 \\
neidade & T1 ou T2 & 7 & 0,31 & 0,33 & 0,25 & 0,33 & 0,03 & 0,211 \\
\hline
\end{tabular}

Em relação do grau de diferenciação, para cada uma das expressões testou-se a hipótese nula de que tumores pouco diferenciados têm resultados iguais aos moderadamente diferenciados e bem diferenciados, versus a hipótese alternativa de resultados diferentes. Na Tabela 5 são apresentados os valores de média, mediana, mínimo, máximo e desvio padrão em relação ao grau de diferenciação.

Os resultados dos testes estatísticos indicaram que não há diferença significativa entre tumores pouco diferenciados e os moderadamente e bem diferenciados, em relação aos índices de marcagem, densidade ótica e heterogeneidade.

\section{Envolvimento nodal}

TABELA 5 - Expressão do Fator VIII e o grau de diferenciação dos tumores

\begin{tabular}{cccccccccc}
\hline Fator VIII & Tipo & n & Média & Mediana Mínimo & Máximo & $\begin{array}{c}\text { Desvio- } \\
\text { padrão }\end{array}$ & $\begin{array}{c}\text { Valor } \\
\text { de } \boldsymbol{P}\end{array}$ \\
\hline $\begin{array}{c}\text { Índice de } \\
\text { marcagem }\end{array}$ & Pouco difer & 6 & 62,67 & 69,55 & 40,55 & 72,61 & 13,45 & 0,701 \\
& Moder ou bem difer & 13 & 64,45 & 71,21 & 29,16 & 75,71 & 13,91 & \\
$\begin{array}{c}\text { Densidade } \\
\text { óptica }\end{array}$ & Pouco difer & 6 & 59,46 & 61,86 & 46,37 & 69,63 & 8,02 & \\
& Moder ou bem difer & 13 & 63,37 & 65,71 & 48,68 & 72,73 & 7,51 & 0,210 \\
$\begin{array}{c}\text { Heteroge- } \\
\text { neidade }\end{array}$ & Pouco difer & 6 & 0,31 & 0,31 & 0,28 & 0,33 & 0,02 & \\
\hline
\end{tabular}

Para cada uma das expressões, testou-se a hipótese nula de que tumores com envolvimento nodal N0 têm resultados iguais aos com envolvimento nodal N1, N2 ou N3, versus a hipótese alternativa de resultados diferentes. Na Tabela 6 são apresentados os valores de média, mediana, mínimo, máximo e desvio-padrão para tumores com envolvimento nodal N0 e os com N1, N2 e N3.

Os resultados dos testes estatísticos indicaram que não TABELA 6 - Expressão do Fator VIII e o envolvimento nodal

\begin{tabular}{cccccccccc}
\hline Fator VIII & Envolv nodal & n & Média & Mediana & Mínimo & Máximo & $\begin{array}{c}\text { Desvio- } \\
\text { padrão }\end{array}$ & $\begin{array}{c}\text { Valor } \\
\text { de } \boldsymbol{P}\end{array}$ \\
\hline $\begin{array}{c}\text { Índice de } \\
\text { marcagem }\end{array}$ & N0 & 7 & 60,86 & 69,15 & 40,55 & 72,81 & 13,63 & 0 \\
& N1, N2 ou N3 & 13 & 65,36 & 71,33 & 29,16 & 75,71 & 13,03 & 0,393 \\
$\begin{array}{c}\text { Densidade } \\
\text { óptica }\end{array}$ & N0 & 7 & 62,40 & 69,63 & 46,37 & 72,73 & 10,96 & \\
& N1, N2 ou N3 & 13 & 61,90 & 62,61 & 48,68 & 67,44 & 5,33 & 0,485 \\
$\begin{array}{c}\text { Heteroge- } \\
\text { neidade }\end{array}$ & N0 & 7 & 0,32 & 0,33 & 0,29 & 0,33 & 0,02 & \\
\hline
\end{tabular}

há diferença significativa entre tumores com envolvimento nodal N0 e os com N1, N2 e N3, em relação aos índices de marcagem, densidade ótica e heterogeneidade.

\section{Padrão histológico}

Para cada uma das expressões, testou-se a hipótese nula de que tumores do tipo intestinal têm resultados iguais ao difuso, versus a hipótese alternativa de resultados diferentes. Na Tabela 7 são apresentados os valores de média, mediana, mínimo, máximo e desvio padrão para tumores do tipo intestinal e difuso.

Os resultados dos testes estatísticos indicaram que não

TABELA 7 - Expressão do Fator VIII e o padrão histológico

\begin{tabular}{ccccccccc}
\hline Fator VIII & Histologia & n & Média & Mediana & Mínimo & Máximo & $\begin{array}{c}\text { Desvio- } \\
\text { padrão }\end{array}$ & $\begin{array}{c}\text { Valor } \\
\text { de } \boldsymbol{P}\end{array}$ \\
\hline $\begin{array}{c}\text { Índice de } \\
\text { marcagem }\end{array}$ & Intestinal & 9 & 63,12 & 71,20 & 29,16 & 74,11 & 15,36 & 1 \\
& Difuso & 11 & 64,33 & 71,21 & 40,55 & 75,71 & 11,61 & 1 \\
$\begin{array}{c}\text { Densidade } \\
\text { óptica }\end{array}$ & Intestinal & 9 & 62,81 & 65,71 & 48,68 & 71,27 & 7,98 & 0,603 \\
$\begin{array}{c}\text { Heteroge- } \\
\text { neidade }\end{array}$ & Difuso & 11 & 61,47 & 61,99 & 46,37 & 72,73 & 7,38 & \\
\hline
\end{tabular}

há diferença significativa entre tumores do tipo intestinal e difuso, em relação aos índices de marcagem, densidade óptica e heterogeneidade.

\section{Idade do paciente}


Para cada uma das expressões dos marcadores, testouse a hipótese nula de que tumores de pacientes com idade de até 65 anos têm resultados iguais aos com mais de 65 , versus a hipótese alternativa de resultados diferentes. $\mathrm{Na}$ Tabela 8 são apresentados os valores de média, mediana, mínimo, máximo e desvio-padrão para tumores de pacientes com idade até 65 anos e acima

Os resultados dos testes estatísticos indicaram que não

TABELA 8 - Expressão do Fator VIII e a idade dos pacientes até 65 e com mais de 65 anos

\begin{tabular}{cccccccccc}
\hline Fator VIII & Idade & n & Média & Mediana & Mínimo & Máximo & $\begin{array}{c}\text { Desvio- } \\
\text { padrão }\end{array}$ & $\begin{array}{c}\text { Valor } \\
\text { de } \boldsymbol{P}\end{array}$ \\
\hline Índice de & Até 65 anos & 11 & 60,91 & 71,21 & 29,16 & 72,81 & 14,47 & 0,370 \\
marcagem & Mais de 65 anos & 9 & 67,30 & 71,20 & 40,55 & 75,71 & 10,89 & \\
$\begin{array}{c}\text { Densidade } \\
\text { óptica }\end{array}$ & Até 65 anos & 11 & 61,45 & 62,61 & 48,68 & 72,73 & 8,00 & \\
Mais de 65 anos & 9 & 62,84 & 62,72 & 46,37 & 71,27 & 7,19 & 0,824 \\
$\begin{array}{c}\text { Heteroge- } \\
\text { neidade }\end{array}$ & Até 65 anos & 11 & 0,31 & 0,32 & 0,25 & 0,35 & 0,03 & \\
\hline
\end{tabular}

há diferença significativa entre tumores de pacientes com idade até 65 anos e os acima, em relação aos índices de marcagem, densidade ótica e heterogeneidade.

\section{DISCUSSÃO}

$\mathrm{O}$ avanço da biologia molecular em tumores tem despertado grande interesse dos pesquisadores em áreas multidisciplinares, com finalidade de detectar o câncer em sua fase precoce, de avaliar o prognóstico e definir a conduta terapêutica. As técnicas de imunodiagnóstico contribuíram para identificação de elementos importantes no ciclo celular e podem ser marcados e identificados.

A identificação de proteínas celulares que estão envolvidas na angiogênese permitiram a evolução na cirurgia, na patologia e no tratamento coadjuvante do câncer gástrico. Contudo, maior entendimento nas alterações moleculares se faz necessário ${ }^{5}$.

A citofotometria de imagem surgiu com o objetivo de obter resultados mais detalhados quando comparados aos de análise da imagem obtida pelo olho humano. A identificação e a quantificação dos marcadores tumorais têm sido avaliadas por análise computadorizada da imagem histológica e utiliza um software instalado em hardware potente para a interpretação da imagem obtida no microscópico óptico. Ela permite medida rápida e acurada, oferecendo grande variedade de aplicações e acessos na biologia, fisiologia e patologia celular. Uma de suas maiores vantagens é a possibilidade de coletar e interpretar grande quantidade de dados ligados à informação morfológica ou estrutural nos tecidos e células. E diversos sistemas comerciais de análise de imagem por computador foram desenvolvidos, como o sistema SAMBA4000, utilizado neste estudo, e desenvolvido com o intuito de minimizar estes fatores de interpretação, objetivando quantificar e qualificar os fenômenos biológicos.

A citofotometria de imagem possibilita, com a imunoistoquímica, identificar os marcadores relacionados ao comportamento biológico das neoplasias, com coloração das estruturas transformando as áreas marcadas em imagens numéricas que são quantificadas pelo computador.

Na obtenção das amostras aqui estudadas estavam registradas as informações dos serviços de anatomia patológica de onde vieram, ou seja, tratava-se de adenocarcinoma gástrico com os dados relacionados. Mesmo assim, cada amostra foi pré-selecionada de acordo com o estado de conservação, confecção e que representavam melhor a massa tumoral. As amostras foram confirmadas pela coloração hematoxilinaeosina e ocorreu grande perda do material parafinado, devido à qualidade ruim que apresentavam tendo que ser excluídos do trabalho. Na primeira seleção do material, separou 55 blocos de parafina, mas apenas 21 tiveram condições técnicas perfeitas para compor a amostra, ou seja, obteve-se perda de $61,82 \%$.

O perfil epidemiológico dos pacientes do presente estudo é semelhante ao divulgado pelo $\mathrm{INCA}^{9}$, no que se refere à predominância dos pacientes do sexo masculino $(61,90 \%)$ e idade superior a 50 anos (idade média de 65 anos). $\mathrm{O}$ fator idade é sempre questionado na evolução e na gravidade da evolução da neoplasia maligna. O que pode ser observado neste estudo dividindo em duas faixas etárias, maior e menor de 65 anos, a expressão do marcador não mostrou diferença significativa com ambos.

No estudo da angiogênese com Fator VIII, Erenoglu et al. ${ }^{6}$, não encontraram correlação entre densidade microvascular e idade ou sexo dos pacientes no câncer gástrico.

Dos marcadores que analisam a evolução do câncer gástrico os que representam a proliferação celular ${ }^{8}$ e a angiogênese $\mathrm{e}^{19}$ parecem ser os melhores. Atualmente os métodos de avaliação da angiogênese é a quantificação de densidade microvascular dos tumores utilizando marcadores específicos para células endoteliais como Fator VIII, CD34, CD31 e VEGF. Quanto à angiogênese no câncer gástrico poderia ser utilizada para indicar, no pré-operatório, os pacientes que se beneficiariam com a quimioterapia neoadjuvante ou tratamento anti-angiogênico.

Aqui neste estudo os tumores apresentaram-se todos avançados com a maioria em T3 ou T4, e quanto mais avançado o tumor, maior angiogênese pode ocorrer em relação à proliferação celular. Segundo estudo de Aotake et al. ${ }^{1}$ na progressão tumoral (displasia a câncer) ocorre aumento da angiogênese que foi observado através da densidade microvascular e com correlação inversa à apoptose; e por sua vez a apoptose tem correlação inversa com a proliferação celular.

Em relação aos parâmetros de medida, o índice de marcagem é o principal. Os demais, como densidade óptica e heterogeneidade que são computados automaticamente são utilizados como parâmetros para avaliar a qualidade do exame. O índice de marcagem, que descreve a quantidade em percentagem da área tecidual que foi corada como um todo, é o sempre citado em pesquisas ${ }^{10,14}$. O marcador Fator VIII apresentou-se como bom indicador de angiogênese.

O estadiamento permanece o mais importante fator determinante do prognóstico para sobrevida em cinco anos e do índice de recorrência local. A importância da profundidade no qual o tumor penetra na parede gástrica, tipo histológico e a presença ou ausência de metástases para linfonodos regionais 
ou órgãos distantes, predizem a cura e a sobrevida.

Tumores com classificação III ou IV apresentaram índice de marcagem maiores do que os com Bormann I ou II, com diferença significativa, ou seja, quanto mais avançado o tumor o Fator VIII tem sua expressão aumentada Porém não se encontrou correlação com a profundidade de invasão tumoral, grau de diferenciação, envolvimento nodal e padrão histológico. O estudo realizado por Erenoglu et al. ${ }^{6}$ em 57 pacientes com câncer gástrico encontrou correlação entre densidade microvascular e parâmetros como tipo difuso e indiferenciado, invasão de serosa e linfonodal, estadio avançado e metástase distal, nos quais se apresentaram com maior densidade vascular e maiores índices, afetando a sobrevida adversamente. Os autores concluiram que a densidade microvascular no carcinoma gástrico pode ser fator prognóstico valioso para predizer pacientes com alto risco para recorrência e para decidir terapia adjuvante pós-operatória. TANIGAWA et al. (1996) em seu estudo com carcinoma gástrico com anti-Fator VIII e anti-CD34 mostrou que existe variabilidade na coloração e contagem vascular entre os dois marcadores, com significativo aumento na expressão de pacientes que apresentaram metástase hematogênica ou peritoneal após procedimento cirúrgico em comparação com aqueles que não apresentaram metástase. Contudo, não houve correlação entre a contagem vascular e metástase linfonodal.

Controvérsias permanecem e escassos trabalhos existem em câncer gástrico com marcadores. Estudos futuros devem correlacionar o Fator VIII com outros marcadores em número maior de pacientes. Dessa forma poder-se-á contribuir mais com o avanço na estratégia médica de combate ao câncer.

\section{CONCLUSÕES}

O presente estudo identificou e marcou $95,24 \%$ das amostras para o Fator VIII. Em relação aos fatores prognósticos, não houve correlação significativa exceto entre o Fator VIII e a classificação de Bormann na qual o tipo III ou IV apresentou maior expressão que o tipo I ou II.

Nakamura MT, Malafaia O, Cuenca RM, Czeczko NG, Cunha RM, Cavalcanti TCS, Ribas CAPM, Czeczko AEA. Cytophotometric study of the expression of the tumoral marker Factor VIII and prognostic factors in gastrci adenocarcinoma. ABCD Arq Bras Cir Dig 2007;20(4):234-40.

ABSTRACT - Introduction - Regarding gastric cancer, the incidence, diagnosis and therapeutic options showed improvement in the last decades, but prognosis remains gloomy, specially due the fact that most patients, already diagnosed present advanced tumors, metastatic and not liable to be surgically resected. Molecular biology is an area in science, which can give the answer to many questions and current scientific facts show that the this should be through detection of tumoral markers. The great advances in informatics refined cell image analysis by image cytophotometry makes it possible to study cell proliferation and angiogenesis in various tumor processes using immunohistochemistry and several markers. At present, studies are conducted to demonstrate the prognostic value of their expressions, however, in gastric adenocarcinoma the results have been divergent and studies are scarce. Aim - To identify and quantify the expression of cell proliferation markers using Ki-67 and of angiogenesis with Factor VIII in gastric adenocarcinoma using cytophotometry, and compare their expressions with factors such as Bormanns' classification, tumor invasion depth, degree of differentiation, nodal involvement, histologic pattern and age. Methods - Twenty-one patients with gastric adenocarcinoma identified between 1998 and 2006 were studied. Ki-67 and Factor VIII expressions were performed using immunohistochemistry with clone MIB-1 primary antibodies, monoclonal for Ki-67 and policlonal for Factor VIII. Cytophotometric analysis was performed using the SAMBA 4000 system. Results - Of the 21 patients $61.90 \%$ were males and $38.10 \%$ females, with a median age of 65 years. In our study eight patients had no Ki-67 marking (61,90\% marking) and only one had no Factor VIII marking (95,24\% marking). Means of Ki-67 labelling index was 33,25\% (standard deviation 20,08, varying from 5,43 to 75,10) and of Factor VIII, $61,14 \%$ (standard deviation 15,06, varying from 29,16 to 73.91). There was no correlation between the two markers regarding gender and age. When compared between the two markers, expression for angiogenesis was significantly greater than that for cell proliferation with a mean difference between labelling index of 20,89 ( $<<0,001)$ and 15,26 standard deviation. There was no correlation between Ki-67 labelling index with comparative factors. However, regarding Factor VIII, tumors classified as III or IV present a significantly greater labelling index than those with Bormann I or II, but there was no correlation with tumor invasion depth, differentiation degree, nodal involvement and histologic pattern. Conclusions - $61,90 \%$ of the samples were identified and marked by Ki-67 and 95,24\% by Factor VIII. In the marked tumors the mean Ki-67 labelling index was 33,25\% and that of the Factor VIII, $61,14 \%$. In the comparative study between the two markers, angiogenesis expression was significantly greater than that of cell proliferation. Regarding prognostic factors there was no significant correlation, except for Factor VIII and Bormanns' classification in which type III or IV was greater than type I or II.

HEADINGS - Gastric adenocarcinoma; Ki-67; Factor VIII; Image cytophotometry; SAMBA.

\section{REFERÊNCIAS}

1. Aotake T, Lu C, Chiba Y, Muraok R, Tanigawa N. Changes of angiogenesis and tumor cell apoptosis during colorectal carcinogenesis. Clinical Cancer Research, v.5, p.135-142, 1999.

2. Catalano V, Labianca R, Beretta GD, Gatta G, De Braud F, Van Cutsem E. Gastric cancer. Critical Reviews in Oncology-Hematology, v.54, n.3, p.209-41, 2005. Revisão.

3. Correa P. Is gastric cancer preventable? Gut, v.53, p.1217-1219, 2004

4. Cotran O. Patologia estrutural e functional. 6.ed., 2000.

5. Dicken BJ, Bigam DL, Cass C, Mackey JR, Joy AA, Hamilton SM. Gastric adenocarcinoma: Review and considerations for future directions. Annals of Surgery, v.241, n.1, p.27-39, 2005.
6. Erenoglu C, Akin ML, Uluutku H, Tezcan L, Yildirim S, Batkin A. Angiogenesis predicts poor prognostic in gastric carcinoma. Digestive Surgery, v.17, n.6, p.581-586, 2000

7. Folkman J. Medicina Interna,15. ed., McGraw Hill, Rio de Janeiro, p.549-62, 2002.

8. Hoang C, Polivka M, Maragi JA, Valleur P, Nemeth J, Galian A. Immunohistochemical detection of cell proliferation in gastric carcinomas with the monoclonal antibody Ki-67. Histology \& Histopathology, v.8, n. 1, p.149-153, 1993.

9. Instituto Nacional de Câncer. Disponível em: $<$ http://www.inca.gov.br $>$ acesso em: 23 fev.2007. 
10. Jesionek KD, Tenderenda M, Rutkowski P. Extent of spontaneous apoptosis in gastric cancer: relation to proliferative index, p53 expression, CD 34 expression and histopathological features. Journal of Experimental \& Clinical Cancer Research, v.21, n.3, p.371-5, 2002.

11. Kimura K, Watanabe H. The diagnosis and treatment of early cancer. GUT, v.41, n. 45, p.13E, 1997.

12. Lauren PA, Nevalainen TJ. Epidemiology of intestinal and diffuse types of gastric carcinoma. A time-trend study in Finland with comparison between studies from high- and low-risk areas. Cancer, v.71, n.10, p.2926-33, 1993

13. Lim L, Michael M, Mann GB, Leong T. Adjuvant therapy in gastric cancer. Journal of Clinical Oncology, v.23, n.25, p.6220-6232, 2005.

14. Manzoni G, Verlato G, Tomezzoli A, Guglielmi A, Pelosi G, Ricci F, Di Leo A, Cordiano C. Study on Ki-67 immunoreactivity as a prognostic indicator in patients with advanced gastric cancer. Japanese Journal of Clinical Oncology, v.28, n.9, p.534-537, 1998.

15. Poon RT, Fan S, Wong J. Clinical implications of circulating angiogenic fac- tors in cancer patients. Journal of Clinical Oncology, v.19, n.4, p.1207-1225, 2001.

16. Rosai J. Ackerman's surgical pathology. 8.ed., 1996.

17. Sayegh ME, Sano T, Dexter S, Katai H, Fukagawa T, Sasako M. TNM and Japanese staging systems for gastric cancer: how do they coexist? Gastric Cancer, v.7, n.3, p.140-8, 2004.

18. Sun W, Haller DG. Recent advances in the treatment of gastric cancer. Drugs, v.61, n.11, p.1545-1551, 2001.

19. Tanigawa N, Amaya H, Matsumura M, Shimomatsuya T, Horiuchi T, Muraoka R, Iki M. Extent of tumor vascularization correlates with prognosis and hematogenous metastasis in gastric carcinomas. Cancer Research, v.56, n.11, p.2671-6, 1996

Conflito de interesse: não há

Fonte financiadora: não há

Recebido para publicação em: 02/06/2007

Aceito para publicação em: 10/08/2007 